\title{
Wave-driven countercurrent plasma centrifuge
}

\author{
Abraham J Fetterman and Nathaniel J Fisch \\ Department of Astrophysical Sciences, Princeton University, Princeton, NJ 08540, USA \\ Received 18 March 2009, in final form 4 June 2009 \\ Published 31 July 2009 \\ Online at stacks.iop.org/PSST/18/045003
}

\begin{abstract}
A method for driving rotation and a countercurrent flow in a fully ionized plasma centrifuge is described. The rotation is produced by radiofrequency waves near the cyclotron resonance. The wave energy is transferred into potential energy in a manner similar to the $\alpha$ channeling effect. The countercurrent flow may also be driven by radiofrequency waves. By driving both the rotation and the flow pattern using waves instead of electrodes, physical and engineering issues may be avoided.
\end{abstract}

(Some figures in this article are in colour only in the electronic version)

Rotating plasmas have been investigated in a variety of configurations for the separation of elements and isotopes [1-4]. Isotope separation has applications in the production of nuclear fuel [5], and in research and medical isotope production [6]. While most enriched uranium is produced by the gas centrifuge method [7], a significant number of isotopes are still produced by calutrons due to their flexibility [8].

The idea to use rotating plasma to separate isotopes was first suggested by Bonnevier, who performed one of the first experiments to test this theory $[1,9]$. In preliminary experiments, significant separation was measured, but it was found that the rotation rate (and therefore separation) was limited by the Alfven critical ionization velocity at the insulator surface along the field lines. The Alfven critical ionization velocity is $v_{\mathrm{c}}=\sqrt{2 e V_{\mathrm{i}} / m}$ where $V_{\mathrm{i}}$ is the ionization potential of the neutral species. Although the mechanism is not entirely understood, attempts to exceed this rotation speed at the insulator surface only result in ablation and increased ionization [1,10-12]. Further experiments revealed that viscous heating fundamentally limits the separation factor in partially ionized plasmas [2].

Both of these limitations were overcome by the introduction of the vacuum-arc plasma centrifuge [3]. These pulsed devices were fully ionized, and did not have enough contact with the wall to be limited by the Alfven critical ionization velocity. However, due to their pulsed nature, throughput is limited, and the concurrent flow pattern limits the separation factor and flexibility of vacuum-arc plasma centrifuges.

The primary method for producing the radial electric field of rotating plasmas is by segmented ring electrodes at the end of the centrifuge [13]. However, the Alfven critical ionization velocity limits the speed of plasma rotation at the electrode surface, even if the plasma is fully ionized [10,13]. The ionization and density must also be carefully controlled to maintain an electrical connection to the end electrodes [14]. Since both the product and waste exit along the field line, these electrodes will be subject to a significant particle flux. A mechanism for removing product and waste from and around the electrodes would be advantageous. It has also been found that some elements of interest, such as uranium, react strongly with electrode materials [15]. These issues suggest that an electrodeless configuration would significantly reduce the engineering difficulty of a plasma centrifuge.

In this paper, we will describe such an electrodeless plasma centrifuge. First we will summarize advantages of the plasma centrifuge over the gas centrifuge. Next, we will give an overview of the device design and its operating parameters. Then we will describe techniques to generate the radial electric field and the countercurrent flow using radiofrequency waves.

\section{Comparison with gas centrifuge}

The separation in a centrifuge (plasma or gas) is produced by the force balance between the pressure and centrifugal forces. Since the centrifugal force is different for each species, there will be different pressure profiles. For a constant rotation frequency $\Omega$, the equilibrium profile is

$$
\left(n_{2} / n_{1}\right)_{r}=\left(n_{2} / n_{1}\right)_{0} \exp \left(\Delta m \Omega^{2} r^{2} / 2 T_{\mathrm{i}}\right),
$$

where $\Delta m=m_{2}-m_{1}$ is the mass difference between the isotopes or elements being separated, which are assumed to 
have equal charge states. In gas centrifuges, the peripheral speed is limited by the stress tolerance of the rotating shell, and so only limited values of the local separation factor $\alpha_{0}=$ $\exp \left(\Delta m \Omega^{2} r^{2} / 2 T_{\mathrm{i}}\right)$ can be achieved. However, in plasmas there is no such limitation.

In order to compare separation methods, we can consider three quantities to be the most important: the single stage separation factor $\alpha$, the single stage separative power $\delta U$ and the energy cost per unit separative power [6]. If the fraction of the desired isotope in the waste of a separation element is $x$ and in the product is $y$, the separation factor is $\alpha=y(1-x) / x(1-y)$. This quantity in general is independent of the concentration of the desired isotope in the feed. The separative power $\delta U$ is the change in $F \phi(z)$ between the input and output streams, where $F$ is the flow rate, $\phi(z)$ is the separative potential, $\phi(z)=(2 z-1) \ln (z /(1-z))$, and $z$ is the concentration of the desired isotope [16].

A plasma centrifuge with length $L$, rotation frequency $\Omega$, column radius $a$ and constant temperature $T$ has the maximum separative power, by analogy with gas centrifuges [16],

$$
\delta U_{\max }=2 \pi\left\langle n D_{\perp}\right\rangle L\left(\frac{\Delta m \Omega^{2} a^{2}}{2 T}\right)^{2},
$$

where the average value for the density times diffusion coefficient $\left\langle n D_{\perp}\right\rangle$ has been used. If we assume that $n$ is independent of radius for most of the separation region, we find $n D_{\perp} \approx T_{\mathrm{i}} n \tau_{\mathrm{i}} \kappa^{2} / m_{\mathrm{i}}(1+\kappa)^{2}$, with $\tau_{\mathrm{i}}$ being the ion collision time and $\kappa=1 /\left(\Omega_{\mathrm{i}} \tau_{\mathrm{i}}\right)$ assumed constant. If we compare this separative power with gas centrifuges, $n D_{\text {gas }}=\sqrt{T_{\text {gas }} / m} / 3 \sigma$, $T_{\text {gas }}=300 \mathrm{~K}, \sigma \approx 5 \times 10^{-15} \mathrm{~cm}^{2}[9]$,

$$
\frac{\delta U_{\text {plasma }}}{\delta U_{\text {gas }}} \approx \frac{T_{\mathrm{i}}^{1 / 2}}{\lambda / 10} \frac{\kappa^{2}}{1+\kappa^{2}}\left(\frac{\Omega a}{10 v_{a}}\right)^{4},
$$

where $T_{\mathrm{i}}$ is in $\mathrm{eV}, v_{a}$ is the peripheral speed of the gas centrifuge and $\lambda$ is the $\log$ of the Debye number.

Since the maximum $v_{a}$ for gas centrifuges is around $500 \mathrm{~m} \mathrm{~s}^{-1}$, a plasma centrifuge will be comparable to a gas centrifuge in separative power if $\Omega a \approx 5 \mathrm{~km} \mathrm{~s}^{-1}$. Vacuumarc centrifuges have achieved up to $\Omega a=10 \mathrm{~km} \mathrm{~s}^{-1}$ for $4 \mathrm{~cm}$ radius columns at $T_{\mathrm{i}}$ around $1 \mathrm{eV}$ [17]. Devices with large radius using hydrogen have regularly achieved rotation speeds of $100 \mathrm{~km} \mathrm{~s}^{-1}\left(T_{\mathrm{i}} \sim 30 \mathrm{eV}\right.$ [18]). In a singular case speeds of $2000 \mathrm{~km} \mathrm{~s}^{-1}$ were achieved $\left(T_{\mathrm{i}} \sim 40 \mathrm{keV} \mathrm{[19]).}\right.$

For our example configuration, $\Omega=4.6 \times 10^{4} \mathrm{~s}^{-1}$, $a=50 \mathrm{~cm}, n=10^{13} \mathrm{~cm}^{-3}, T_{\mathrm{i}}=10 \mathrm{eV}, L=10 \mathrm{~m}$, the device would produce $1.6 \times 10^{-3} \mathrm{~mol} \mathrm{SWU} \mathrm{s}^{-1}$ (separative work units/second). The estimated power usage for the waves is $57 \mathrm{~kW}$, so that the total energy cost is $370 \mathrm{eV} \mathrm{SWU}^{-1}$. This may be compared with $650 \mathrm{eV} \mathrm{SWU}^{-1}$ for gas centrifuges [5]. Although this reduction seems marginal (especially since there are certainly other loss mechanisms not accounted for), 97\% of the cost of separation by gas centrifuge is due to capital costs for construction and operation [7]. In this sense, the plasma centrifuge represents a significant reduction in cost. A single gas centrifuge has a separative power of $1.2 \times$ $10^{-6} \mathrm{~mol} \mathrm{SWU} \mathrm{s}^{-1}$, so that the described plasma centrifuge may replace a cascade of 1300 gas centrifuges.

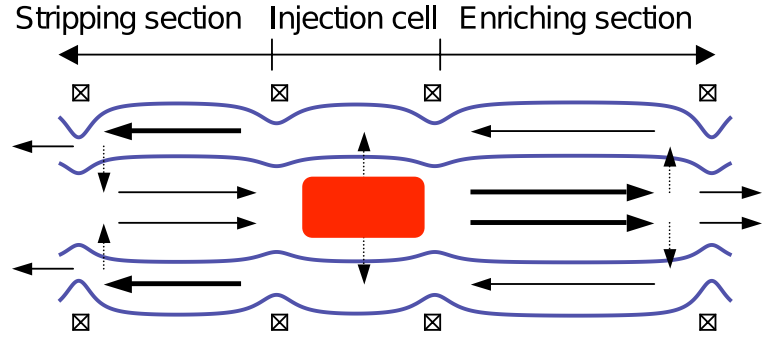

Figure 1. An example of the countercurrent plasma centrifuge (symmetric about the center axis). The solid lines indicate field lines, the shaded region is where the plasma is created, the solid arrows indicate flow patterns and the dashed arrows indicate wave-induced diffusion.

Table 1. Frequencies and lengths.

\begin{tabular}{ll}
\hline$\Omega_{\mathrm{i}}=4.1 \times 10^{4} \mathrm{~s}^{-1}$ & $a=50 \mathrm{~cm}$ \\
$\tilde{\Omega}_{\mathrm{i}}=1.3 \times 10^{5} \mathrm{~s}^{-1}$ & $\rho_{\mathrm{i}}=1.5 \mathrm{~cm}$ \\
$\Omega_{E}=10^{5} \mathrm{~s}^{-1}$ & $L=1000 \mathrm{~cm}$ \\
$\Omega=4.6 \times 10^{4} \mathrm{~s}^{-1}$ & $\lambda_{\mathrm{i}}=20 \mathrm{~cm}$ \\
$\nu_{\mathrm{i}}=10^{4} \mathrm{~s}^{-1}$ & \\
\hline
\end{tabular}

\section{Overview of design}

The proposed device is a multiple-mirror configuration, with at least two magnetic mirrors. An example is shown in figure 1. The plasma is created in a central cell near the axis of the device. The ions are diffused outward radially to produce the rotation. Countercurrent flow patterns are then created in the enriching and stripping sections, with multiple-mirror cells used as needed to control the flow pattern. The product stream recovering the light element is removed on the right, and the waste stream containing the heavy element is removed on the left.

In order to facilitate discussion of different parameters, and to present a unified picture of a workable device, we will use a consistent set of parameters, although different designs could vary by an order of magnitude. We consider an axial magnetic field $B=0.1 \mathrm{~T}$, a plasma column of radius $a=50 \mathrm{~cm}$ and length $L=10 \mathrm{~m}$ with constant density $n=10^{13} \mathrm{~cm}^{-3}$ and temperature $T=10 \mathrm{eV}$. The electric field providing the rotation will have magnitude $E=50 \mathrm{~V} \mathrm{~cm}^{-1}$ at $r=a$. The species we will consider separating are ${ }^{235} \mathrm{U}$ and ${ }^{238} \mathrm{U}$. The related frequencies and lengths are shown in table 1.

This design will describe how to create a plasma centrifuge assuming that the elements or isotopes to be separated have a similar ratio of mass to charge $m / q$. As such, both species will be treated as a single fluid. Once the rotation is created and flow patterns are known, the exact separation may be determined by the differential equation [20],

$$
\begin{aligned}
n \frac{\partial x}{\partial t}= & \frac{1}{r} \frac{\partial}{\partial r}\left(n D_{\perp}\left[r \frac{\partial x}{\partial r}+x(1-x) \frac{\Delta m \Omega^{2} r^{2}}{T}\right]\right) \\
& -n V_{z} \frac{\partial x}{\partial z}+n D_{\|} \frac{\partial^{2} x}{\partial z^{2}} .
\end{aligned}
$$

where $x$ is the density fraction that is species $i$ and $\Delta m=$ $m_{j}-m_{i}$.

The rotation is produced by a radial current in the injection cell. The $\boldsymbol{J} \times \boldsymbol{B}$ force creates the rotation and maintains 
it against losses due to ion-neutral collisions. The current maintains the radial electric potential, $\Phi \approx(m / 2 q) \Omega_{\mathrm{i}} \Omega_{E} r^{2}$, with $\Omega_{\mathrm{i}}$ the cyclotron frequency and $\Omega_{E}=-E / r B$ the $E \times B$ rotation frequency. The true rotation frequency will differ from $\Omega_{E}$ due to the centrifugal force. Ignoring the diamagnetic drift, the rotation frequency is given by

$$
\Omega=\frac{\Omega_{\mathrm{i}}}{2}\left(\sqrt{1+4 \frac{\Omega_{E}}{\Omega_{\mathrm{i}}}}-1\right) .
$$

In order to maximize the throughput of the device, the density must be as large as possible. The density will be proportional to the dimensionless ratio $\kappa=v_{\mathrm{i}} / \Omega_{\mathrm{i}}$. This parameter $\kappa$ is limited by requirements for resonant waveparticle interactions. However, since $\lambda_{\mathrm{i}} \ll L$, viscosity and angular momentum transport are strong and we will assume that the plasma is rigidly rotating (that is, the rotation frequency is independent of radius). We will also require that the stripping and enriching sections are several mean-free-paths long, so that the plasma has a local Maxwellian distribution. The length of the injection cell will be determined by requirements for the ionization of injected neutrals.

Two new technologies are involved. First, a method is described to produce the required rotation within the injection cell (rather than at the end electrodes) using waves at the ion cyclotron resonance. Second, we show how a countercurrent flow pattern may be created using waves at the ion Landau resonance near a mirror boundary.

\section{Rotation}

The use of electrodes to drive plasma rotation faces many obstacles. The Alfven critical ionization velocity must be avoided, unreactive electrode materials must be chosen to withstand extreme environments and the plasma density and ionization must be carefully controlled to maintain electrical contact. These issues provide a strong argument for alternative methods of driving plasma rotation [15].

It was shown previously that the energy of alpha particles from fusion reactions can provide the necessary energy for plasma rotation, with the exchange mediated by appropriate radiofrequency waves [21]. It is possible to use the same technique to convert wave energy to potential energy without a kinetic energy source. This wave may be launched in the injection cell to maintain the rotation against the drag of neutrals in that chamber. If the neutral density is low in the remainder of the device, the drag in those regions will also be low.

We consider a wave that has lab frame frequency $\omega$, parallel wave number $k_{\|}$and poloidal mode number $n_{\theta}$. The rotating frame wave frequency $\tilde{\omega}=\omega-n_{\theta} \Omega$ is resonant with the $n$th rotating frame cyclotron harmonic of a particle with parallel velocity $v_{\|}$, so that $\tilde{\omega}-k_{\|} v_{\|}=n \tilde{\Omega}_{\mathrm{i}}$. We note that the rotating frame cyclotron frequency is modified by the Coriolis force, so that $\tilde{\Omega}_{\mathrm{i}}=\Omega_{\mathrm{i}}+2 \Omega$ [22]. Rotating frame quantities are denoted here with a tilde.

The interaction of the wave with the particle will create a correlated change in its rotating frame perpendicular kinetic

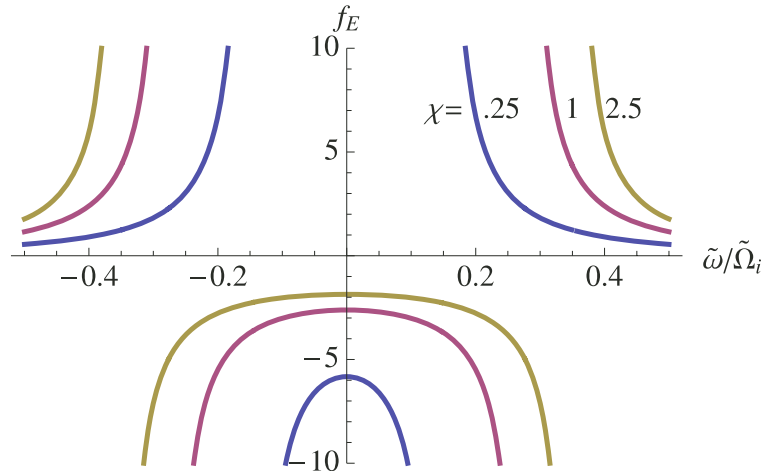

Figure 2. The branching ratio $f_{E}$ versus $\tilde{\omega} / \tilde{\Omega}_{\mathrm{i}}$ for $n_{\theta}=-1$ and $\chi=\Omega_{E} / \Omega_{\mathrm{i}}=0.25,1$ and 2.5 .

energy $\tilde{W}_{\perp}$ and radial position, $r \Delta r=\Delta \tilde{W}_{\perp} n_{\theta} /\left(m \tilde{\omega} \tilde{\Omega}_{\mathrm{i}}\right)$ [23]. Since there is a radial electric field, the change in radial position corresponds to a change in electric potential energy, $q \Delta \Phi=-q E \Delta r=n_{\theta}\left(\Omega_{E} \Omega_{\mathrm{i}} / \tilde{\omega} \tilde{\Omega}_{\mathrm{i}}\right) \Delta \tilde{W}_{\perp}$. There is also a correlated change in the parallel kinetic energy, $\Delta W_{\|}=$ $\left(k_{\|} v_{\|} / n \tilde{\Omega}_{\mathrm{c}}\right) \Delta \tilde{W}_{\perp}$.

The fraction of the kinetic energy that is converted into potential energy was defined as the branching ratio, which is [21],

$$
\begin{aligned}
f_{E} & =\frac{-n_{\theta} \Omega_{E} \Omega_{\mathrm{i}}}{\omega \tilde{\Omega}_{\mathrm{i}}+\tilde{\omega} k_{\|} v_{\|} / n-n_{\theta} \Omega_{E} \Omega_{\mathrm{i}}}, \\
& =\frac{-n_{\theta} \chi}{(1+4 \chi)\left(\tilde{\omega} / \tilde{\Omega}_{\mathrm{i}}\right)^{2}+\left(n_{\theta} / 4\right)(\sqrt{1+4 \chi}-1)^{2}} .
\end{aligned}
$$

where $\chi=\Omega_{E} / \Omega_{\mathrm{i}}$. For the purpose of driving rotation without a significant kinetic energy source, the potential energy must be converted from wave energy. This sets the condition $\left|f_{E}\right| \gg 1$. In mirrors, waves with low mode numbers $n_{\theta}$ have been coupled into the plasma [24-26]. Large values of $\left|f_{E}\right|$ are accessible for low mode numbers at low frequencies, $\tilde{\omega} / \tilde{\Omega}_{\mathrm{i}} \ll 1$ (see figure 2 ). Negative values of $f_{E}$ correspond to the rest-frame ion energy increasing as the particle moves out radially. However, in the rotating frame the ion energy will decrease with increasing radius as long as $n_{\theta} / \tilde{\omega}<0$. The reason for this discrepancy is that the wave azimuthal phase velocity $\tilde{\omega} / k_{\theta}$ changes directions between the rest and rotating frames.

Because the collision frequency is significant, we cannot depend on phase space manipulations to result in nonMaxwellian distributions lasting more than one bounce time, as was done in the case of alpha channeling. But we do, as in alpha channeling in collisionless plasmas, rely on the fact that ions will be more likely to move down density gradients in phase space. This requires that there is a limit to the motion in one direction, and that there is a density gradient along the diffusion path. The limitation in energy may be accomplished if the particle decreases in energy as it moves out $\left(n_{\theta}<0\right)$. Then the density must decrease fast enough as the radius increases to maintain a population inversion along the diffusion path. Assuming a constant Maxwellian temperature profile, we may 


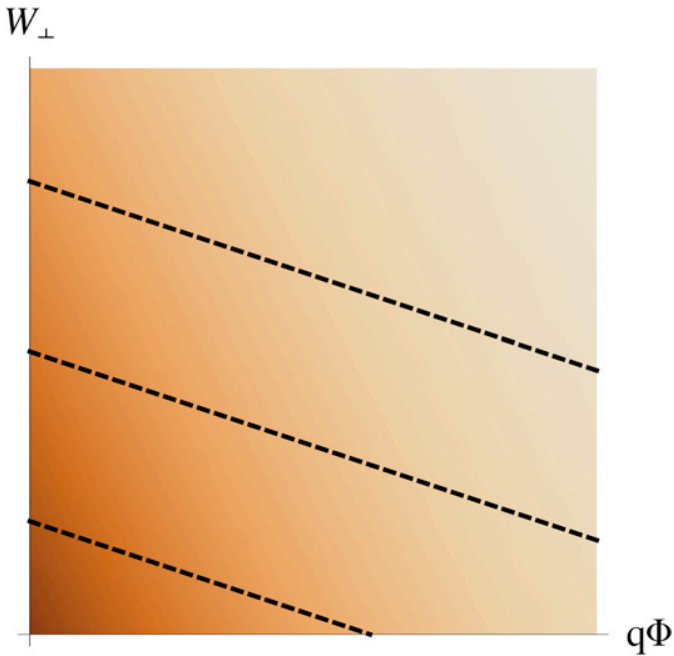

Figure 3. Phase space density plot with diffusion paths to drive rotation drawn (dashed lines). The $x$-axis variable is related to the radial coordinate by $q \Phi \propto r^{2}$. The temperature everywhere is constant.

write this condition,

$$
\begin{aligned}
& 0>\Delta\left(n \mathrm{e}^{-\tilde{W} / T}\right), \\
& 0>\frac{\partial n}{\partial r} \Delta r \mathrm{e}^{-\tilde{W} / T}-\frac{\Delta \tilde{W}_{\perp}+\Delta W_{\|}}{T} n \mathrm{e}^{-\tilde{W} / T} .
\end{aligned}
$$

Using the equation for the change in parallel energy and radius, we find the condition,

$$
\frac{\partial \ln n}{\partial r}<\frac{m \tilde{\omega}^{2} r}{n_{\theta} T} .
$$

For $n_{\theta}<0$, this yields a minimum steepness for the radial density gradient. Integrating, one finds that the maximum radius of a Gaussian density distribution is $r_{\max }=\left(\tilde{\Omega}_{\mathrm{i}} / \tilde{\omega}\right) \rho_{\mathrm{i}}$. Because of this, we should choose a frequency $\tilde{\omega}$ as small as possible, even if the efficiency $f_{E}$ is sacrificed. Example diffusion paths are shown in figure 3 . As the ions diffuse down the density gradient, they move outward, producing a $\boldsymbol{J} \times \boldsymbol{B}$ force that sustains the plasma rotation. Most of the energy consumed in this process is provided by the wave, although some energy may be absorbed or provided by the ions (by heating or cooling, depending on the sign of $f_{E}$ ).

An upper bound for the power requirements for this wave may be found by noting that the radial potential is the source of most thermal and rotation energy. Thus, for flow rate $G$, the wave would require at least the power $G \Omega^{2} r_{0}^{2} / 4$, assuming a constant density profile from 0 to $r_{0}$ and $\frac{1}{2} m \Omega^{2} r_{0}^{2} \gg T$. If uranium is processed at $0.4 \mathrm{~g} \mathrm{~s}^{-1}$, the wave producing the rotation would require $52 \mathrm{~kW}$ of power. This number may be reduced if rotation energy is recovered when the particle is removed, for example by using a shaped collector [27].

\section{Countercurrent production}

It is clear that, if possible, countercurrent operation is desirable, since the separative effect can be multiplied many times in one device. Bonnevier suggested that the parallel flow may be driven by placing a conductor along the centrifuge axis, and driving a current along this conductor to create a poloidal magnetic field. A parallel velocity gradient would then be created by shear in the $B_{\theta} E_{r} / B^{2}$ drift [9]. This method relies on inserting a conductor into the plasma confinement region. Our method instead uses waves to provide parallel momentum to the plasma at selected radii. This momentum balances the viscous damping of the countercurrent flow pattern.

Momentum can be provided to the ions by using waves with parallel phase velocities near the thermal velocity of the ions. The resonance condition is $\tilde{\omega}-k_{\|} v_{\|}=0$. Since for the Maxwellian particle distribution function (PDF) $f$, $\partial f / \partial v<0$, more particles will be accelerated by this wave than are damped. The result is a flattening in the tail of the Maxwellian distribution (see figure 5). This creates net parallel force acting on the plasma as the Maxwellian plasma passes through the wave region.

Because the plasma is collisional, this wave must be placed within an ion mean free path $\lambda_{\mathrm{i}}$ of the mirror point (see figure 4). It is the reflection condition at the mirror that will create a radially varying force profile. The reflection condition is, for mirror ratio $R_{\mathrm{m}}$,

$$
W_{\|}<W_{\perp}\left(R_{\mathrm{m}}-1\right)+\frac{1}{2} m \Omega^{2} r^{2}\left(1-R_{\mathrm{m}}^{-1}\right) .
$$

In the case $R_{\mathrm{m}}-1 \ll 1$, this may be written $v_{\|}<$ $\Omega r \sqrt{1-R_{\mathrm{m}}^{-1}}$. So as the radius increases, the cutoff velocity for reflection increases linearly. It will be helpful to define a scaled radius $r^{\prime}=\Omega r \sqrt{1-R_{\mathrm{m}}^{-1}}$, with units of velocity. If we suppose that there is an identical plasma on the other side of the mirror with $\operatorname{PDF} f$, and the PDF modified by the Landau resonance is $f^{\prime}$, then defining $g=f^{\prime}-f$ the force produced by the wave after reflection, at scaled radius $r^{\prime}$ is

$$
\begin{aligned}
F\left(r^{\prime}\right)= & \frac{2 \pi r^{\prime}}{\Omega \sqrt{1-R_{\mathrm{m}}^{-1}}} \int_{r^{\prime}}^{\infty} g\left(v_{\|}\right) m v_{\|} v_{\|} \mathrm{d} v_{\|} \\
& -\frac{2 \pi r^{\prime}}{\Omega \sqrt{1-R_{\mathrm{m}}^{-1}}} \int_{-\infty}^{r^{\prime}} g\left(v_{\|}\right) m v_{\|} v_{\|} \mathrm{d} v_{\|} .
\end{aligned}
$$

In the integral we multiply the momentum $m v_{\|}$by the velocity $v_{\|}$of particles passing through the wave surface and integrate over the PDF. The result can be seen in figure 5. At small radii, all the resonant particles pass through the mirror, so the net force is positive. At $r^{\prime}=v_{\| \text {res }}$, there is a maximum in the force, as particles that lost energy are reflected and particles that gained energy are transmitted through the mirror. For large radii, all resonant particles are reflected, so the net force is in the reverse direction.

We can also estimate the magnitude of this force. If we consider that $N$ particles that are pushed an average of $\Delta v$ near resonance $v_{\text {res }}$, such that $\partial f^{\prime}\left(v_{\text {res }}\right) / \partial v=0$, we find $N=n_{0}\left(v_{\text {res }}(\Delta v)^{2} / v_{\text {th }}^{3}\right) \mathrm{e}^{-v_{\text {res }}^{2} / 2 v_{\text {th }}^{2}}$. These particles have parallel velocity approximately $v_{\text {res }}$, so the force is $F=m \Delta v v_{\text {res }} N$. At radii $\left|r^{\prime}-v_{\text {res }}\right|<\Delta v / 2$, the particles shifted by $\Delta v$ have a net change in velocity of $2 v_{\text {res }}$ since they are transmitted rather than reflected. Thus, the magnitude of the force integrated over the radius is, after some simplification,

$$
F_{\text {rf }}=\pi a^{2} n_{0} m v_{\text {res }}^{2} c_{\text {res }} \frac{(\Delta v)^{3}}{v_{\text {th }}^{3}} \mathrm{e}^{-v_{\text {res }}^{2} / 2 v_{\text {th }}^{2}}
$$



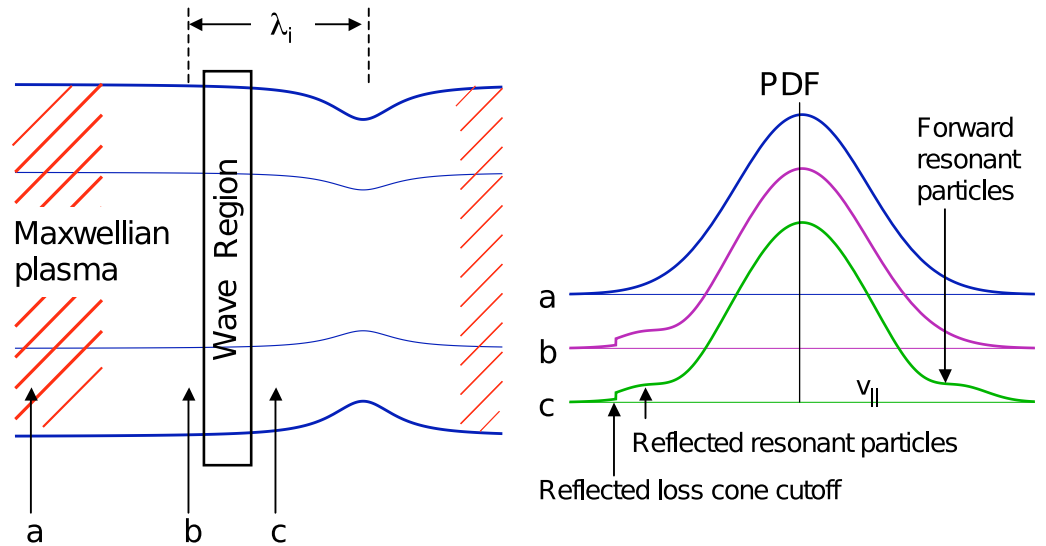

Figure 4. Left: diagram of wave region and mirror throat. Solid lines indicate field lines. The boxed area is the region for wave-particle interaction. Right: particle distribution functions at locations $\mathrm{a}, \mathrm{b}$ and $\mathrm{c}$ on the left diagram.

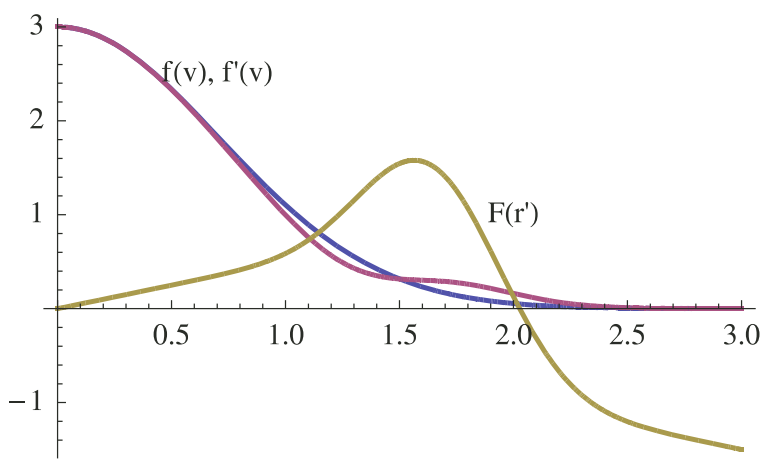

Figure 5. The particle distribution function and parallel force. The Maxwellian PDF and the PDF modified by a Landau resonance at $v_{\|}=1.5 v_{\text {th }}$ are plotted versus parallel velocity in units of $v_{\text {th }}$. The parallel force is plotted versus radius, with radius in units of $v_{\mathrm{th}} /\left(\Omega \sqrt{1-R_{\mathrm{m}}^{-1}}\right)$. Vertical units are arbitrary.

where $c_{\text {res }}=1+4 v_{\text {res }}^{2} /\left(\Omega^{2} a^{2}\left(1-R_{\mathrm{m}}^{-1}\right)\right)$ is a constant taking into account the special acceleration at the resonant radius.

The force due to the viscosity is, for constant density and temperature [28],

$$
m n_{0} \frac{\partial V_{z}}{\partial t}=1.2 \frac{n_{0} T v_{\mathrm{i}}}{\Omega_{\mathrm{i}}^{2}} \frac{1}{r} \frac{\partial}{\partial r}\left(r \frac{\partial V_{z}}{\partial r}\right) .
$$

If $V_{z}=V_{z 0}+r V_{z 1} / a$ for $r=0$ to $a$,

$$
m n_{0} \frac{\partial V_{z}}{\partial t}=2.4 \frac{n_{0} T v_{\mathrm{i}}}{\tilde{\Omega}_{\mathrm{i}}^{2}} \frac{V_{z 1}}{r a},
$$

or integrating over the volume,

$$
F_{\text {vis }}=5 \pi n_{0} m v_{\mathrm{th}}^{2} V_{z 1} \frac{v_{\mathrm{i}}}{\tilde{\Omega}_{\mathrm{i}}^{2}} L .
$$

Setting $F_{\text {rf }}=F_{\text {vis }}$ to find out the steady state $V_{z 1}$,

$$
V_{z 1}=v_{\text {th }} \frac{c_{\text {res }}}{5} \frac{\lambda_{\mathrm{i}}}{L} \frac{a^{2}}{\rho_{\mathrm{i}}^{2}} \frac{v_{\mathrm{res}}^{2}}{v_{\text {th }}^{2}} \frac{(\Delta v)^{3}}{v_{\text {th }}^{3}} \mathrm{e}^{-v_{\text {res }}^{2} / 2 v_{\text {th }}^{2}} .
$$

We want to compare this velocity with the optimal value. The maximum for the separation fraction occurs when the total internal flux $\int_{0}^{a}\left|n V_{z}\right| r \mathrm{~d} r=2 \sqrt{2} \pi a n_{0} v_{\text {th }} \rho_{\mathrm{i}} N_{\mathrm{f}}$ [20], where $N_{\mathrm{f}}$ is the flux index dependent only on the flow profile. In this case the optimum recirculating velocity is $V_{z 1}^{\star}=20 \sqrt{2} v_{\text {th }}\left(\rho_{\mathrm{i}} / a\right) N_{\mathrm{f}}$. The ratio of these two quantities is

$$
\frac{V_{z 1}}{V_{z 1}^{\star}}=\frac{c_{\text {res }}}{c_{\text {nf }}} \frac{\lambda_{\mathrm{i}}}{L} \frac{a^{3}}{\rho_{\mathrm{i}}^{3}} \frac{v_{\mathrm{res}}^{2}}{v_{\mathrm{th}}^{2}} \frac{(\Delta v)^{3}}{v_{\mathrm{th}}^{3}} \mathrm{e}^{-v_{\mathrm{res}}^{2} / 2 v_{\mathrm{th}}^{2}},
$$

where we have folded $c_{\mathrm{nf}}=100 \sqrt{2} N_{\mathrm{f}}$ into one variable describing the flow shape. We can find the necessary value for the width of the resonance $\Delta v$ using this equation,

$$
\frac{(\Delta v)^{3}}{v_{\text {th }}^{3}}=\frac{c_{\text {nf }}}{c_{\text {res }}} \frac{V_{z 1}}{V_{z 1}^{\star}} \frac{L}{\lambda_{\mathrm{i}}} \frac{\rho_{\mathrm{i}}^{3}}{a^{3}} \frac{v_{\mathrm{th}}^{2}}{v_{\text {res }}^{2}} \mathrm{e}^{v_{\text {res }}^{2} / 2 v_{\text {th }}^{2}} .
$$

We can now estimate the power used by this wave. $N$ particles change energy by $m v_{\text {res }} \Delta v$, and they flow through the wave region with velocity $v_{\text {res }}$. Therefore,

$$
\begin{aligned}
P & =\pi a^{2} m n_{0} v_{\mathrm{res}}^{3} \frac{(\Delta v)^{3}}{v_{\mathrm{th}}^{3}} \mathrm{e}^{-v_{\mathrm{res}}^{2} / 2 v_{\mathrm{th}}^{2}} \\
& =\pi a^{2} n_{0} T v_{\mathrm{res}} \frac{c_{\mathrm{nf}}}{c_{\mathrm{res}}} \frac{V_{z 1}}{V_{z 1}^{\star}} \frac{L}{\lambda_{\mathrm{i}}} \frac{\rho_{\mathrm{i}}^{3}}{a^{3}} .
\end{aligned}
$$

Note the strong dependence on $\rho_{\mathrm{i}} / a$, the only term that is significantly less than one, allowing cancellation of the large shape factor in $c_{\mathrm{nf}}$. For the proposed configuration, (assuming $\left.V_{z 1}=V_{z 1}^{\star}\right), 5 \mathrm{~kW}$ of power are required for a $10 \mathrm{~m}$ long plasma column.

Here we have discussed waves that will drive the main countercurrent flow. Other waves may also be required to enhance radial transport near mirror boundaries other than at the injection cell (see figure 1). This radial transport may be done using waves of the same type described in the rotation section. Waves may also be needed to enhance the removal of product or waste from the trap. Either waves described here, Alfven ion cyclotron waves [29], or other waves known to cause ion 'pump-out' [30], may be used for this purpose.

\section{Conclusion}

We have described a countercurrent plasma centrifuge that is entirely driven by radiofrequency waves. Because the plasma 
is confined by the magnetic field, it is not limited in its rotation velocity. As such, much higher separation factors and separative power may be achieved than in traditional gas centrifuges.

By driving the rotation using waves, the device avoids the requirement for end electrodes to drive rotation. The end electrodes tended to incur many issues in past devices $[11,13]$. They may limit the rotation speed to the Alfven critical ionization velocity. They also can react strongly with elements to be separated such as uranium [2]. Finally, they are physically in the way of removing the product and waste of the separation, and so may present many other engineering challenges.

The operation of the plasma centrifuge in a countercurrent has been suggested before [9], and equations describing the separation along the axis for a fully ionized countercurrent plasma centrifuge have been derived [20]. Countercurrent operation is critical to an efficient separation scheme and allows an increased separation factor without multiple stages. The countercurrent is produced in the device suggested here without a center conductor. The use of waves gives precise control of the countercurrent force along the plasma axis. This will allow increased efficiency of the separation process, as the flow can be tailored to approximate an ideal cascade [5].

This theoretical work only lays the conceptual foundation for this type of device. It remains to identify waves that can be coupled into a rotating plasma and that might accomplish these effects. The kind of waves necessary to couple into the rotating plasma centrifuge will be very similar to the kind of waves necessary for coupling into magnetic mirror machines for the purposes of extracting energy from the alpha particles [31, 32]. While low azimuthal-mode-number waves have been coupled to stationary mirrors before [24-26], not much is known about coupling waves to plasmas with supersonic rotation. In addition, a method must be found to inject neutrals into the core of the rotating plasma to be ionized, so that the density profile is peaked there. This allows the radial diffusion of ions to be bounded and favorable.

\section{Acknowledgments}

This work was supported by DOE Contracts DE-FG0206ER54851 and DE-AC0276-CH03073.

\section{References}

[1] Bonnevier B 1971 Experimental evidence of element and isotope separation in a rotating plasma Plasma Phys. 13763

[2] Wijnakker M M B and Granneman E H A 1980 Limitations on mass separation by the weakly ionized plasma centrifuge Z. Naturf. a 35 883-93

[3] Krishnan M, Geva M and Hirschfield J L 1981 Plasma centrifuge Phys. Rev. Lett. 46 36-8

[4] Ohkawa T and Miller R 2002 Band gap ion mass filter Phys. Plasmas 95116

[5] Benedict M 1981 Nucl. Chem. Eng. (New york: McGraw-Hill)

[6] Grossman M W and Shepp T A 1991 Plasma isotope-separation methods IEEE Trans. Plasma Sci. 19 1114-22

[7] United States Gas Centrifuge Program for Uranium Enrichment 1977 Technical Report US Department of Energy
[8] Tracy J and Terry J 1985 Availability of enriched isotopic materials used for accelerator targets-present and future Nucl. Instrum. Methods Phys. Res. Section B: Beam Interact. Mater. Atoms 10-11 972-5

[9] Bonnevier B 1967 Diffusion due to ion-ion collisions in a multicomponent plasma Ark. Fys. 33255

[10] Alfvén H 1960 Collision between a nonionized gas and a magnetized plasma Rev. Mod. Phys. 32 710-3

[11] Bergstrom J 1976 Experiments on the velocity distribution of a rotating plasma Nucl. Instrum. Methods 133 347-53

[12] Lai S T 2001 A review of critical ionization velocity Rev. Geophys. 39 471-506

[13] Lehnert B 1971 Rotating plasmas Nucl. Fusion 11485

[14] Bekhtenev A A and Volosov V I 1978 Generation of a radial electric field in a rotating plasma Sov. Phys.: Tech. Phys. $\mathbf{2 3}$ $938-41$

[15] Witalis E A 1981 Electrodeless plasma isotope centrifuge Atomkernenerg. Kerntech. $\mathbf{3 8} 32$

[16] Cohen K 1951 The Theory of Isotope Separation as Applied to the Large Scale Production of $U-235$ (New york: McGraw-Hill)

[17] Krishnan M 1983 Centrifugal isotope separation in zirconium plasmas Phys. Fluids 262676

[18] Ellis R F, Case A, Elton R, Ghosh J, Griem H, Hassam A B, Lunsford R A, Messer S J and Teodorescu C 2005 Steady supersonically rotating plasmas in the maryland centrifugal experiment Phys. Plasmas 1255704

[19] Abdrashitov G F, Beloborodov A V, Volosov V I, Kubarev V V, Popov Yu S and Yudin Yu N 1991 Hot rotating plasma in the psp-2 experiment Nucl. Fusion 311275

[20] Ivanov A A and Timchenko N N 1990 Counterstreaming mass separation of ions in a fully ionized rotating plasma Sov. J. Plasma Phys. 16 863-6

[21] Fetterman A J and Fisch N J 2008 Alpha channeling in a rotating plasma Phys. Rev. Lett. 101205003

[22] Bonnevier B and Lehnert B 1960 The motion of charged particles in a rotating plasma Ark. Fys. 16 231-6

[23] Fisch N J and Rax J M 1992 Interaction of energetic alpha particles with intense lower hybrid waves Phys. Rev. Lett. $69612-5$

[24] Amagishi Y and Inutake M 1982 Conversion of compressional alfvén waves into ion-cyclotron waves in inhomogeneous magnetic fields Phys. Rev. Lett. 48 1183-6

[25] Majeski R, Browning J J, Meassick S, Hershkowitz N, Intrator T and Ferron J R 1987 Effect of variable eigenmode excitation on rf stabilization of a mirror plasma Phys. Rev. Lett. 59 206-9

[26] Yamaguchi Y, Ichimura M, Higaki H, Kakimoto S, Nakagome K, Nemoto K, Katano M, Nakajima H, Fukuyama A and Cho T 2006 Eigenmode formation of ICRF waves in gamma 10 Plasma Phys. Control. Fusion 481155

[27] Bekhtenev A A, Volosov V I, Bal'chikov V E, Pekker M S and Yardin Yu N 1980 Problems of a thermonuclear reactor with a rotating plasma Nucl. Fusion 20 579-97

[28] Braginskii S I 1965 Transport processes in a plasma Rev. Plasma Phys. 1 205-311

[29] Goto T, Ishii K, Goi Y, Kikuno N, Katsuki Y, Yamanashi M, Nakamura M, Ichimura M, Tamano T and Yatsu K 2000 Ion diffusion in a velocity space induced by alfvén ion cyclotron mode observed in a mirror plasma Phys. Plasmas 72485

[30] Baldwin D E 1977 End-loss processes from mirror machines Rev. Mod. Phys. 49 317-39

[31] Fisch N J 2006 Alpha channeling in mirror machines Phys. Rev. Lett. 97225001

[32] Zhmoginov A I and Fisch N J 2008 Simulation of $\alpha$-channeling in mirror machines Phys. Plasmas 15042506 